MATEMATIKA, 2017, Volume 33, Number 1, 71-85

(C) Penerbit UTM Press. All rights reserved

\title{
Some generating functions involving certain polynomials of two variables
}

\author{
${ }^{1}$ Ahmed Ali Al-Gonah and ${ }^{2}$ Hussein Abdulhafed Saleh \\ ${ }^{1,2}$ Department of Mathematics, Aden University, Aden, Yemen \\ e-mail: ${ }^{1}$ gonah1977@yahoo.com
}

\begin{abstract}
In the present paper, we derive families of bilateral and mixed multilateral generating relations involving two variables Hermite and two variables extended Srivastava polynomials. Further, as applications of the main results, several bilateral and trilateral generating functions involving certain polynomials of two variables are obtained by using some recent known generating functions.
\end{abstract}

Keywords Generating functions; Hermite polynomials; extended Srivastava polynomials.

2010 Mathematics Subject Classification 33B10, 33C45, 33E30.

\section{Introduction}

Let $\left\{A_{n, k}\right\}_{n, k=0}^{\infty}$ be a bounded double sequence of real or complex numbers. Over four decades ago, Srivastava [1] introduced the Srivastava polynomials (SP) $S_{n}^{N}(x)$ by the following series definition:

$$
S_{n}^{N}(x)=\sum_{k=0}^{\left[\frac{n}{N}\right]} \frac{(-n)_{N k}}{k !} A_{n, k} x^{k}\left(n \in \mathbb{N}_{0}=\mathbb{N} \cup\{0\} ; N \in \mathbb{N}\right),
$$

where $\mathbb{N}$ is the set of positive integer $[a]$ denotes the greatest integer in $a \in \mathbb{R}$ and $(\lambda)_{v},(\lambda)_{0} \equiv 1$, denotes the Pochhammer symbol defined in terms of familiar Gamma function by $[2$, p. 22]

$$
(\lambda)_{v}=\frac{\Gamma(\lambda+v)}{\Gamma(\lambda)}=\left\{\begin{array}{ll}
1 & (v=0 ; \lambda \in \mathbb{C} \backslash\{0\}) \\
\lambda(\lambda+1)(\lambda+2) \cdots(\lambda+n-1) & (v=n \in \mathbb{N} ; \lambda \in \mathbb{C})
\end{array} .\right.
$$

The Srivastava polynomials $S_{n}^{N}(x)$ in (1.1) and their such interesting variants as follows:

$$
S_{n, q}^{N}(x)=\sum_{k=0}^{\left[\frac{n}{N}\right]} \frac{(-n)_{N k}}{k !} A_{n+q, k} x^{k}\left(q, n \in \mathbb{N}_{0} ; N \in \mathbb{N}\right),
$$

were investigated rather extensively in $[3]$. See $[4,5]$ for further results. Clearly we have

$$
S_{n, 0}^{N}(x)=S_{n}^{N}(x) .
$$

Motivated essentially by the definitions (1.2) and (1.3), the following family of the two variables extended Srivastava polynomials (2VESP) $S_{n}^{q, N}(x, y)$ was introduced and studied by Altin et al. [6]:

$$
S_{n}^{q, N}(x, y)=\sum_{k=0}^{\left[\frac{n}{N}\right]} A_{q+n, k} \frac{x^{n-N k} y^{k}}{(n-N k) ! k !}\left(q, n \in \mathbb{N}_{0} ; N \in \mathbb{N}\right)
$$


and further studied in [7].

However, in view of definitions (1.3) and (1.5), it is easily observed that

$$
S_{n}^{q, N}(x, y)=\frac{x^{n}}{n !} S_{n, q}^{N}\left(\frac{y}{(-x)^{N}}\right)
$$

which for $x=-1$ we get

$$
S_{n}^{q, N}(-1, y)=\frac{(-1)^{n}}{(n) !} S_{n, q}^{N}(y) .
$$

It has been shown in [6] that the $S_{n}^{q, N}(x, y)$ include many well-known polynomials of two variables such as Lagrange-Hermite polynomials, Lagrange polynomials and two-variables Hermite polynomials, under the special choices for the double sequence $\left\{A_{n, k}\right\}$. Here, we will recall them and add further new particular cases as the following remarks:

Remark 1.1 ([6, p. 316] see also [7, p. 1116]). Choosing

$$
N=1, A_{q, n}=(\alpha)_{q-n}(\beta)_{n}\left(q, n \in \mathbb{N}_{0}\right)
$$

in equation (1.5), we get

$$
S_{n}^{q, 1}(x, y)=(\alpha)_{q} g_{n}^{(\alpha+q, \beta)}(x, y),
$$

where $g_{n}^{(\alpha, \beta)}(x, y)$ denotes the two variable Lagrange polynomials defined by [2 p. 441]

$$
g_{n}^{(\alpha, \beta)}(x, y)=\sum_{k=0}^{n} \frac{(\alpha)_{n-k}(\beta)_{k}}{(n-k) ! k !} x^{n-k} y^{k}
$$

and specified by the following generating function

$$
(1-x t)^{-\alpha}(1-y t)^{-\beta}=\sum_{n=0}^{\infty} g_{n}^{(\alpha, \beta)}(x, y) t^{n} .
$$

Remark 1.2 ([6, p. 316] see also [7, p. 1116]). Choosing

$$
N=2, A_{q, n}=(\alpha)_{q-2 n}(\beta)_{n}\left(q, n \in \mathbb{N}_{0}\right)
$$

in equation(1.5), we get

$$
S_{n}^{q, 2}(x, y)=(\alpha)_{q} h_{n}^{(\alpha+q, \beta)}(x, y),
$$

where $h_{n}^{(\alpha, \beta)}(x, y)$ denotes the Lagrang-Hermite polynomials defined by [8]

$$
h_{n}^{(\alpha, \beta)}(x, y)=\sum_{k=0}^{\left[\frac{n}{2}\right]} \frac{(\alpha)_{n-2 k}(\beta)_{k}}{(n-2 k) ! k !} x^{n-2 k} y^{k}
$$

and specified by the following generating function:

$$
(1-x t)^{-\alpha}\left(1-y t^{2}\right)^{-\beta}=\sum_{n=0}^{\infty} h_{n}^{(\alpha, \beta)}(x, y) t^{n} .
$$


Now, we add the following new particular cases as remarks:

Remark 1.3 Choosing $A_{q, n}=1\left(q, n \in \mathbb{N}_{0}\right)$ in equation (1.5), we get

$$
S_{n}^{q, N}(x, y)=\frac{1}{n !} H_{n}^{(N)}(x, y),
$$

where $H_{n}^{(N)}(x, y)$ denotes the Gould Hopper polynomials (GHP) defined by [9]

$$
H_{n}^{(N)}(x, y)=n ! \sum_{k=0}^{\left[\frac{n}{N}\right]} \frac{x^{n-N k} y^{k}}{(n-N k) ! k !}
$$

and specified by the following generating function:

$$
\exp \left(x t+y t^{N}\right)=\sum_{n=0}^{\infty} H_{n}^{(N)}(x, y) \frac{t^{n}}{n !} .
$$

Note that, for $N=1$ in equation (1.14) and using the relation

$$
H_{n}^{(1)}(x, y)=(x+y)^{n}
$$

we get

$$
S_{n}^{q, 1}(x, y)=\frac{(x+y)^{n}}{n !} .
$$

Also, for $N=2$ in equation (1.14) and using the relation

$$
H_{n}^{(2)}(x, y)=H_{n}(x, y)
$$

we get the known relation $[6$, p. 316]

$$
S_{n}^{q, 2}(x, y)=\frac{1}{n !} H(x, y),
$$

where $H_{n}(x, y)$ denotes the two variables Hermit-Kamp de Friet polynomials (2VHKdFP) defined by $[10$, p. 341]

$$
H_{n}(x, y)=n ! \sum_{k=0}^{\left[\frac{n}{2}\right]} \frac{x^{n-2 k} y^{k}}{(n-2 k) ! k !}
$$

and specified by the following generating function:

$$
\exp \left(x t+y t^{2}\right)=\sum_{n=0}^{\infty} H_{n}(x, y) \frac{t^{n}}{n !} .
$$

Remark 1.4 Choosing $A_{q, n}=1 / n !,\left(q, n \in \mathbb{N}_{0}\right)$ in equation (1.5), we get

$$
S_{n}^{q, N}(y, x)=\frac{1}{n !}{ }_{N} L_{n}(x, y),
$$


where ${ }_{N} L_{n}(x, y)$ denotes the two variables generalized Laguerre polynomial (2VgLP) defined by [11 p. 213]

$$
{ }_{N} L_{n}(x, y)=n ! \sum_{k=0}^{\left[\frac{n}{N}\right]} \frac{x^{k} y^{n-N k}}{(n-N k) !(k !)^{2}}
$$

and specified by the following generating function

$$
\exp (y t) C_{0}\left(-x t^{N}\right)=\sum_{n=0}^{\infty}{ }_{N} L_{n}(x, y) \frac{t^{n}}{n !},
$$

where $C_{0}(x)$ denotes the $0^{\text {th }}$ order Tricomi function. The $n^{\text {th }}$ order Tricomi functions $C_{n}(x)$ are defined by $[2]$

$$
C_{n}(x)=\sum_{k=0}^{\infty} \frac{(-1)^{k} x^{k}}{k !(n+k) !} .
$$

For $N=1$ and $x=-x$ in equation (1.25), the $2 \mathrm{VgLP}_{N} L_{n}(x, y)$ reduce to the two variables Laguerre polynomials $(2 \mathrm{VLP}) L_{n}(x, y)$, i.e., we have

$$
{ }_{1} L_{n}(-x, y)=L_{n}(x, y),
$$

where $L_{n}(x, y)$ defined by [11]:

$$
L_{n}(x y)=n ! \sum_{k=0}^{n} \frac{(-1)^{k} x^{k}}{(n-k) !} \frac{y^{n-k}}{(k !)^{2}}
$$

an specified by the following generating function

$$
\exp (y t) C_{0}(-x t)=\sum_{n=0}^{\infty} L_{n}(x, y) \frac{t^{n}}{n !} .
$$

Note that for $N=1$ in equation (1.23) and using relation (1.27), we get

$$
S_{n}^{q, 1}(y, x)=\frac{1}{n !} L_{n}(-x, y) .
$$

Further for $N=2$ in equation (1.23), we get

$$
S_{n}^{q, 2}(y, x)=\frac{1}{n !}{ }_{2} L_{n}(x, y)=\frac{1}{n !} S_{n}(-x, y),
$$

where $S_{n}(x, y)$ denotes the second form of the two variables Legendre polynomials (2VLeP) defined by $[12]$

$$
S_{n}(x, y)=n ! \sum_{k=0}^{\left[\frac{n}{2}\right]} \frac{(-1)^{k} x^{k} y^{n-2 k}}{(n-2 k) !(k !)^{2}}
$$

an specified by the following generating function

$$
\exp (\mathrm{yt}) C_{0}\left(x t^{2}\right)=\sum_{n=0}^{\infty} S_{n}(x, y) \frac{t^{n}}{n !} .
$$


Remark 1.5 Choosing $N=1, A_{q, n}=\frac{(-1)^{q-n}}{n !(q-n) !},\left(q, n \in N_{0}\right)$ in equation (1.5), we get

$$
S_{n}^{0,1}(x, y)=\frac{R_{n}(x, y)}{(n !)^{2}}
$$

where $R_{n}(x, y)$ denotes the first form of the two variables Legendre polynomials defined by $[13]$

$$
R_{n}(x, y)=(n !)^{2} \sum_{k=0}^{n} \frac{(-1)^{n-k} x^{n-k} y^{k}}{((n-k) !)^{2}(k !)^{2}} .
$$

The aim of this paper is to derive some families of bilateral and mixed multilateral generating relations involving the 2 VHKdFP $H_{n}(x, y)$ and the $2 \operatorname{VESP} S_{n}^{q, N}(x, y)$ by using series rearrangement techniques. Also, the above mentioned remarks will be used to obtain some illustrative bilateral and trilateral generating functions involving the $2 \operatorname{VHKdFP} H_{n}(x, y)$ and many other known two variables polynomials. For this aim we recall the following generating function for the $2 \mathrm{VHKdFP} H_{n}(x, y)[2]$

$$
\sum_{n=0}^{\infty}(\alpha)_{n} H_{n}(x, y) \frac{t^{n}}{n !}=(1-x t)_{2}^{-a} \mathrm{~F}_{0}\left(\frac{\alpha}{2}, \frac{\alpha+1}{2} ;-; \frac{4 y t^{2}}{(1-x t)^{2}}\right) .
$$

The $2 \mathrm{VHKdFP} H_{n}(x, y)$ are linked to the classical Hermite polynomials $H_{n}(x)$ by the relations

$$
H_{n}(x, y)=(-i)^{n} y^{n / 2} H_{n}\left(\frac{i x}{2 \sqrt{y}}\right)
$$

and

$$
H_{n}(2 x,-1)=H_{n}(x),
$$

where the classical Hermite polynomials $H_{n}(x)$ defined by [14, p. 187]

$$
H_{n}(x)=n ! \sum_{k=0}^{\left[\frac{n}{2}\right]} \frac{(-1)^{k}(2 x)^{n-2 k}}{k !(n-2 k) !}
$$

Also, the 2VHKdFP $H_{n}(x y)$ satisfy the following relation:

$$
t^{n} H_{n}(x, y)=H_{n}\left(x t, y t^{2}\right) .
$$

\section{Bilateral generating relations}

We prove the following results:

Theorem 2.1 The following family of bilateral generating relation involving the 2VHKdFP $H_{n}(x, y)$ and the 2VESP $S_{n}^{q, N}(x, y)$ holds true:

$$
\sum_{q, n=0}^{\infty} H_{q+n}(u, v) S_{n}^{q, N}(x, y) \frac{t^{n} w^{q}}{q !}=\sum_{n, q=0}^{\infty} H_{q+N n}(u, v) A_{q+N n, n} \frac{\left(\mathrm{yt}^{N}\right)^{n}}{n !} \frac{(w+x t)^{q}}{q !}
$$


Proof. Using definition (1.5), we have

$$
\text { l.h.s }=\sum_{q, n=0}^{\infty} H_{q+n}(u, v) \sum_{k=0}^{\left[\frac{n}{N}\right]} A_{q+n, k} \frac{x^{n-N k} y^{k}}{(n-N k) ! k !} \frac{t^{n} w^{q}}{q !}
$$

which on replacing $n$ by $n+N k$ in the r.h.s. and using the lemma [2, p. 101]

$$
\sum_{n=0}^{\infty} \sum_{k=0}^{\left[\frac{n}{m}\right]} A(k, n)=\sum_{n=0}^{\infty} \sum_{k=0}^{\infty} A(k, n+m k) .
$$

in the resultant equation becomes

$$
\text { l.h.s }=\sum_{n, q, k=0}^{\infty} H_{n+q+N k}(u, v) A_{n+q+N k, k} \frac{x^{n}}{n !} \frac{y^{k}}{k !} \frac{t^{n+N k} w^{q}}{q !} .
$$

Again replacing $q$ by $q-n$ in the r.h.s. of equation (2.4) and using the lemma [2, p. 100]

$$
\sum_{n=0}^{\infty} \sum_{k=0}^{\infty} A(k, n)=\sum_{n=0}^{\infty} \sum_{k=0}^{n} A(k, n-k)
$$

in the resultant equation, we get

$$
\text { l.h.s }=\sum_{q, k=0}^{\infty} H_{q+N k}(u, v) A_{q+N k, k} \frac{\left(y t^{N}\right)^{k}}{q ! k !} \sum_{n=0}^{q} \frac{q !(x t)^{n} w^{q-n}}{n !(q-n) !} .
$$

Finally using the binomial theorem [2]

$$
(x+y)^{n}=\sum_{k=0}^{n} \frac{n ! x^{n-k} y^{k}}{k !(n-k) !}
$$

in the r.h.s. of the above equation, we get the r.h.s. of equation (2.1).

Remark 2.1. Taking $w=-x t$ in assertion (2.1) of Theorem 2.1, we deduce the following consequence of Theorem 2.1:

Corollary 2.1 The following family of bilateral generating relation involving the 2VHKdFP $H_{n}(x, y)$ and the 2 VESP $S_{n}^{q, N}(x, y)$ holds true:

$$
\sum_{q, n=0}^{\infty} H_{q+n}(u, v) S_{n}^{q, N}(x, y) \frac{(-x)^{q} t^{n+q}}{q !}=\sum_{n=0}^{\infty} H_{N n}(u, v) A_{N n, n} \frac{\left(y t^{N}\right)^{n}}{n !}
$$

Remark 2.2 Taking $w=0$ in assertion (2.1) of Theorem 2.1 we deduce the following consequence of Theorem 2.1:

Corollary 2.2 The following family of bilateral generating relation involving the 2VHKdFP $H_{n}(x, y)$ and the 2 VESP $S_{n}^{q, N}(x, y)$ holds true:

$$
\sum_{n=0}^{\infty} H_{n}(u, v) S_{n}^{0, N}(x, y) t^{n}=\sum_{q, n=0}^{\infty} H_{q+N n}(u, v) A_{q+N n, n} \frac{\left(\mathrm{yt}^{N}\right)^{n}}{n !} \frac{(x t)^{q}}{q !} .
$$

In the next section, Corollaries 2.1 and 2.2 will be exploited to derive families of mixed multilateral generating relations involving the 2VHKdFP $H_{n}(x, y)$ and $2 \mathrm{VESP} S_{n}^{q, N}(x, y)$ with the help of the method considered in [2]. 


\section{Multilateral generating relations}

We prove the following theorem by using Corollary 2.1:

Theorem 3.1 Corresponding to an identically non-vanishing function $\Omega_{\mu}\left(z_{1}, \ldots, z_{l}\right)$ of complex variables $z_{1}, \ldots, z_{l}(l \in \mathbb{N})$ and of complex order $\mu$, let

$$
\Lambda_{\mu, \psi}\left(z_{1}, \ldots, z_{l} ; \eta\right):=\sum_{k=0}^{\infty} a_{k} \Omega_{\mu+\psi k}\left(z_{1}, \ldots, z_{l}\right) \eta^{k}\left(a_{k} \neq 0, \psi, \eta \in \mathbb{C}\right),
$$

then we have, for $n, p \in \mathbb{N}$

$$
\begin{array}{r}
\sum_{q=0}^{\infty} \sum_{n=0}^{\infty} \sum_{k=0}^{\left[\frac{q}{p}\right]} a_{k} H_{q+n-p k}(u, v) S_{n}^{q-p k, N}(x, y) \Omega_{\mu+\psi k}\left(z_{1}, \ldots, z_{l}\right) \eta^{k} \frac{(-x)^{q-p k}(w t)^{q+n-p k}}{(q-p k) !} \\
=\Lambda_{\mu, \psi}\left(\mathrm{z}_{1}, \ldots, \mathrm{z} ; \eta\right) \sum_{n=0}^{\infty} H_{N n}(u, v) A_{N n, n} \frac{\left(\mathrm{y}(w t)^{N}\right)^{n}}{n !}
\end{array}
$$

provided that each member of assertion (3.2) exists.

Proof Using relation (2.3), we find

$$
\text { l.h.s }=\sum_{k=0}^{\infty} a_{k} \Omega_{\mu+\psi k}\left(z_{1}, \ldots, z_{l}\right) \eta^{k} \sum_{q, n=0}^{\infty} H_{q+n}(u, v) S_{n}^{q, N}(x, y) \frac{(-x)^{q}(w t)^{q+n}}{q !}
$$

Using equations (3.1) and (2.8) in the r.h.s. of equation (3.3), we get the r.h.s. of equation (3.2).

Next, following the same procedure leading to assertion (3.2) of Theorem 3.1 and using Corollary 2.2, we get the following result:

Theorem 3.2 Corresponding to an identically non-vanishing function $\Omega_{\mu}\left(z_{1}, \ldots, z_{l}\right)$ of complex variables $z_{1}, \ldots, z_{l}(l \in \mathbb{N})$ and of complex order $\mu$, let

$$
\begin{aligned}
\Lambda_{\mu, \psi}\left(z_{1}, \ldots, z_{l} ; \eta\right) & :=\sum_{k=0}^{\infty} a_{k} \Omega_{\mu+\psi k}\left(z_{1}, \ldots, z_{l}\right) \eta^{k}\left(a_{k} \neq 0, \psi, \eta \in \mathbb{C}\right) \\
\Theta_{n, p}^{\mu, \psi}\left(u, v, x, y ; z_{1}, \ldots, z_{l} ; \tau\right): & =\sum_{k=0}^{\left[\frac{n}{p}\right]} a_{k} H_{n-p k}(u, v) S_{n-p k}^{0, N}(x, y) \Omega_{\mu+\psi k}\left(z_{1}, \ldots, z_{l}\right) \tau^{k}
\end{aligned}
$$

where $n, p \in \mathbb{N}$. Then, we have

$$
\begin{aligned}
\sum_{n=0}^{\infty} \Theta_{n, p}^{\mu, \psi}\left(u, v, x, y, z_{1}, \ldots, z_{l} ; \frac{\eta}{t^{p}}\right) t^{n} & \\
& =\Lambda_{\mu, \psi}\left(z_{1}, \ldots, z_{l} ; \eta\right) \sum_{q, n=0}^{\infty} H_{q+N n}(u, v) A_{q+N n, n} \frac{\left(y t^{N}\right)^{n}}{n !} \frac{(x t)^{q}}{q !} .
\end{aligned}
$$

provided that each member of assertion (3.5) exists. 
Notice that, for every suitable choice of the coefficients $a_{k}\left(k \in \mathbb{N}_{0}\right)$, if the multivariable function $\Omega_{\mu+\psi k}\left(z_{1}, \ldots, z_{l}\right),(l \in \mathbb{N})$, is expressed in terms of simpler function of one and more variables, the assertions of Theorems 3.1 and 3.2 can be applied in order to derive various families of multilinear and multilateral generating relations involving some polynomials of two variables

For example, if we set $l=2, \psi=1,=0, \Omega_{k}\left(z_{1}, z_{2}\right)=L_{k}\left(z_{1}, z_{2}\right)$ and $a_{k}=1 / k$ ! in assertion (3.2) of Theorem 3.1 and using (1.28) we readily obtain the following mixed trilateral generating function:

$$
\begin{gathered}
\sum_{q=0}^{\infty} \sum_{n=0}^{\infty} \sum_{k=0}^{\left[\frac{q}{p}\right]} H_{q+n-p k}(u, v) S_{n}^{q-p k, N}(x, y) L_{k}\left(z_{1} z_{2}\right) \frac{\eta^{k}(-x)^{q-p k}(w t)^{q+n-p k}}{k !(q-p k) !} \\
=\exp \left(z_{2} \eta\right) C_{0}\left(-z_{1} \eta\right) \sum_{n=0}^{\infty} H_{N n}(u, v) A_{N n, n} \frac{\left(y(w t)^{N}\right)^{n}}{n !}
\end{gathered}
$$

In the next section, we derive some bilateral and trilateral generating functions for the 2VHKdFP $H_{n}(x, y)$ and other polynomials as applications of the results derived in Sections 2 and 3 with the help of certain generating functions and the remarks introduced in Section 1.

\section{Applications}

First, the following bilateral generating functions are obtained as applications of Corollaries 2.1 and 2.2 :

(i) Taking $N=1$ and $\left\{A_{q, n}\right\}_{q, n=0}^{\infty}$ as in Remark 1.1 and using relation (1.8) in equation (2.8), we get

$$
\sum_{q, n=0}^{\infty}(\alpha)_{q} H_{q+n}(u, v) g_{n}^{(\alpha+q, \beta)}(x, y) \frac{(-x)^{q} t^{n+q}}{q !}=\sum_{n=0}^{\infty}(\beta)_{n} H(u, v) \frac{(y t)^{n}}{n !}
$$

which on using equation (1.36) in the r.h.s. gives the following bilateral generating function:

$$
\begin{aligned}
\sum_{q, n=0}^{\infty}(\alpha)_{q} H_{q+n}(u, v) g_{n}^{(\alpha+q, \beta)} & (x y) \frac{(-x)^{q} t^{n+q}}{q !} \\
& =(1-u y t)_{2}^{-\beta} F_{0}\left(\frac{\beta}{2}, \frac{\beta+1}{2} ;-; \frac{4 v y^{2} t^{2}}{(1-u y t)^{2}}\right) .
\end{aligned}
$$

Now, using the following relation between the Jacobi polynomials $P_{n}^{(\alpha, \beta)}(x, y)$ and Lagrange polynomials $[2$, p. 442$]$ :

$$
g_{n}^{(\alpha, \beta)}(x, y)=(y-x)^{n} P_{n}^{(-\alpha-n,-\beta-n)}\left(\frac{x+y}{x-y}\right),
$$


in the r.h.s. of equation (4.2), we get

$$
\begin{aligned}
\sum_{q, n=0}^{\infty}(\alpha)_{q} H_{q+n}(u, v) & P_{n}^{(-\alpha-q-n,-\beta-n)}\left(\frac{x+y}{x-y}\right) \frac{(y-x)^{n}(-x)^{q} t^{n+q}}{q !} \\
= & (1-u y t)^{-\beta} 2 F_{0}\left(\frac{\beta}{2}, \frac{\beta+1}{2} ;-; \frac{4 v y^{2} t^{2}}{(1-u y t)^{2}}\right) .
\end{aligned}
$$

Again taking $N=1$ and $\left\{A_{q, n}\right\}_{q, n=0}^{\infty}$ as in Remark 1.1 and using relation (1.8) in equation (2.9), we get

$$
\sum_{n=0}^{\infty} H_{n}(u, v) g_{n}^{(\alpha, \beta)}(x, y) t^{n}=\sum_{q, n=0}^{\infty}(\alpha)_{q}(\beta)_{n} H_{q+n}(u, v) \frac{(\mathrm{yt})^{n}}{n !} \frac{(x t)^{q}}{q !}
$$

which on using relation (4.3) in the r.h.s. becomes

$$
\begin{aligned}
\sum_{n=0}^{\infty} H_{n}(u, v) & P_{n}^{(-\alpha-q-n,-\beta-n)}\left(\frac{x+y}{x-y}\right)(y t-x t)^{n} \\
& =\sum_{q, n=0}^{\infty}(\alpha)_{q}(\beta)_{n} H_{q+n}(u, v) \frac{(\mathrm{yt})^{n}}{n !} \frac{(x t)^{q}}{q !}
\end{aligned}
$$

(ii) Taking $N=2$ and $\left\{A_{q, n}\right\}_{q, n=0}^{\infty}$ as in Remark 1.2 and using relation (1.11) in equation (2.8), we get

$$
\sum_{q, n=0}^{\infty}(\alpha)_{q} H_{q+n}(u, v) h_{n}^{(\alpha+q, \beta)}(x, y) \frac{(-x)^{q} t^{n+q}}{q !}=\sum_{n=0}^{\infty}(\beta)_{n} H_{2 n}(u, v) \frac{\left(\mathrm{yt}^{2}\right)^{n}}{n !} .
$$

Again taking $N=2$ and $\left\{A_{q, n}\right\}_{q, n=0}^{\infty}$ as in Remark 1.2 and using relation (1.11) in equation (2.9), we get

$$
\sum_{n=0}^{\infty} H_{n}(u, v) h_{n}^{(\alpha, \beta)}(x, y) t^{n}=\sum_{q, n=0}^{\infty}(\alpha)_{n}(\beta)_{n} H_{q+2 n}(u, v) \frac{\left(\mathrm{yt}^{2}\right)^{n}}{n !} \frac{(x t)^{q}}{q !} .
$$

(iii) Taking $\left\{A_{q, n}\right\}_{q, n=0}^{\infty}$ as in Remark 1.3 and using relation (1.14) in equation (2.8), we get

$$
\sum_{q, n=0}^{\infty} H_{q+n}(u, v) H_{n}^{(N)}(x, y) \frac{(-x)^{q} t^{n+q}}{n ! q !}=\sum_{n=0}^{\infty} H_{N n}(u, v) \frac{\left(y t^{N}\right)^{n}}{n !}
$$

which for $N=2$ and then using relation (1.19) and the following generating function $[15$, p. 412]:

$$
\frac{1}{\sqrt{1-4 v z}} \exp \left(\frac{u^{2} z}{1-4 v z}\right)=\sum_{n=0}^{\infty} H_{2 n}(u, v) \frac{z^{n}}{n !}
$$

in the l.h.s. and r.h.s. of the resultant equation respectively gives

$$
\sum_{q, n=0}^{\infty} H_{q+n}(u, v) H_{n}(x, y) \frac{(-x)^{q} t^{n+q}}{n ! q !}=\frac{1}{\sqrt{1-4 v y t^{2}}} \exp \left(\frac{u^{2} y t^{2}}{1-4 v y t^{2}}\right) \text {. }
$$


Again taking $\left\{A_{q, n}\right\}_{q, n=0}^{\infty}$ as in Remark 1.3 and using relation (1.14) in equation (2.9), we get

$$
\sum_{n=0}^{\infty} H_{n}(u, v) H_{n}^{(N)}(x, y) \frac{t^{n}}{n !}=\sum_{q, n=0}^{\infty} H_{q+N n} \frac{\left(\mathrm{y} t^{N}\right)^{n}}{n !} \frac{(x t)^{q}}{q !}
$$

which for $N=2$ and using relation (1.19) gives

$$
\sum_{n=0}^{\infty} H_{n}(u, v) H_{n}(x, y) \frac{t^{n}}{n !}=\sum_{q, n=0}^{\infty} H_{q+2 n}(u, v) \frac{\left(\mathrm{y} t^{2}\right)^{n}}{n !} \frac{(x t)^{q}}{q !} .
$$

Now, using the following bilinear generating function [16, p. 717$]$ :

$$
\sum_{n=0} H_{n}(x, y) H_{n}(z, w) \frac{t^{n}}{n !}=\frac{1}{\sqrt{1-4 y t^{2} w}} \exp \left(\frac{\mathrm{xz} t+t^{2}\left(w x^{2}+y z^{2}\right)}{1-4 y t^{2} w}\right)
$$

in the l.h.s. of equation (4.13), we get

$$
\frac{1}{\sqrt{1-4 y t^{2} v}} \exp \left(\frac{x u t+t^{2}\left(v x^{2}+y u^{2}\right)}{1-4 y t^{2} v}\right)=\sum_{q, n=0}^{\infty} H_{q+2 n}(u, v) \frac{\left(y t^{2}\right)^{n}}{n !} \frac{(x t)^{q}}{q !},
$$

which for $x=0$ and $y t^{2}=z$ gives equation (4.10).

(iv) Taking $\left\{A_{q, n}\right\}_{q, n=0}^{\infty}$ as in Remark 1.4 and using relation (1.23) in equation (2.8), we get

$$
\sum_{q, n=0}^{\infty} H_{q+n}(u, v)_{N} L_{n}(x, y) \frac{(-y)^{q} t^{n+q}}{n ! q !}=\sum_{n=0}^{\infty} H_{N n}(u, v) \frac{\left(x t^{N}\right)^{n}}{(n !)^{2}} .
$$

Now, for $N=1$ and replacing $x$ by $-x$ in equation (4.16) and using equation (1.27) we get

$$
\sum_{q, n=0}^{\infty} H_{q+n}(u, v) L_{n}(x, y) \frac{(-y)^{q} t^{n+q}}{n ! q !}=\sum_{n=0} H_{n}(u, v) \frac{(-x t)^{n}}{(n !)^{2}},
$$

which on using relation (1.40) in the r.h.s. and then using the following definition of the $n^{t h}$ order Hermite-Tricomi function [15, p. 407 ]: (for $n=0$ )

$$
{ }_{H} C_{n}(x, y)=\sum_{k=0}^{\infty} \frac{(-1)^{k} H_{k}(x, y)}{k !(k+n) !}
$$

gives the following bilateral generating function:

$$
\sum_{q, n=0}^{\infty} H_{q+n}(u, v) L_{n}(x, y) \frac{(-y)^{q} t^{n+q}}{n ! q !}={ }_{H} C_{0}\left(u x t, v x^{2} t^{2}\right) .
$$

Also for $N=2$ and replacing $x$ by $-x$ in equation(4.16) and using equation (1.31), we get

$$
\sum_{q, n=0}^{\infty} H_{q+n}(u, v) S_{n}(x, y) \frac{(-y)^{q} t^{n+q}}{n ! q !}=\sum_{n=0}^{\infty} H_{2 n}(u, v) \frac{\left(-x t^{2}\right)^{n}}{(n !)^{2}}
$$


which on using relation (1.40) in the r.h.s. and then using the following definition of the $0^{\text {th }}$ order Hermite-Bessel functions [12]

$$
{ }_{H} J_{0}(x, y)=\sum_{r=0}^{\infty} \frac{(-1)^{r} H_{2 r}(x, y)}{2^{2 r}(r !)^{2}}
$$

gives the following bilateral generating function:

$$
\sum_{q, n=0}^{\infty} H_{q+n}(u, v) S_{n}(x, y) \frac{(-y)^{q} t^{n+q}}{n ! q !}={ }_{H} J_{0}\left(2 u \sqrt{x} t, 4 v x t^{2}\right) .
$$

Again taking $\left\{A_{q, n}\right\}_{q, n=0}^{\infty}$ as in Remark 1.4 and using relation (1.23) in equation (2.9), we get

$$
\sum_{n=0}^{\infty} H_{n}(u, v)_{N} L_{n}(x, y) \frac{t^{n}}{n !}=\sum_{q, n=0}^{\infty} H_{q+N n}(u, v) \frac{\left(x t^{N}\right)^{n}}{(n !)^{2}} \frac{(y t)^{q}}{q !}
$$

which for $N=1$ and replacing $x$ by $-x$ and using equation (1.27) becomes

$$
\sum_{n=0}^{\infty} H_{n}(u, v) L_{n}(x, y) \frac{t^{n}}{n !}=\sum_{q, n=0}^{\infty} H_{q+n}(u, v) \frac{(-x t)^{n}}{(n !)^{2}} \frac{(y t)^{q}}{q !} .
$$

Now, using the following bilateral generating function $[16$, p. 718$]$ :

$$
\sum_{n=0}^{\infty} H_{n}(x, y) L_{n}(z, w) \frac{t^{n}}{n !}=\exp \left(y(w t)^{2}+x w t\right){ }_{H} C_{0}\left(2 z t\left(\frac{x}{2}+y w t\right), y(z t)^{2}\right)
$$

in the l.h.s. of equation (4.24), we get

$$
\begin{aligned}
\exp \left(v(y t)^{2}+u y t\right){ }_{H} C_{0} & \left(2 x t\left(\frac{u}{2}+v y t\right), v(\mathrm{x} t)^{2}\right) \\
= & \sum_{q, n=0}^{\infty} H_{q+n}(u, v) \frac{(-x \mathrm{t})^{n}}{(n !)^{2}} \frac{(y t)^{q}}{q !} .
\end{aligned}
$$

Also for $N=2$ in equation (4.23), we get

$$
\sum_{n=0}^{\infty} H_{n}(u, v) L_{n}(x, y) \frac{t^{n}}{n !}=\sum_{q, n=0}^{\infty} H_{q+2 n}(u, v) \frac{\left(x t^{2}\right)^{n}}{(n !)^{2}} \frac{(y t)^{q}}{q !}
$$

which on using the following bilateral generating function $[16$, p. 720$]$ :

$$
\begin{aligned}
\sum_{n=0}^{\infty} H_{n}(x, y){ }_{2} L_{n}(z, w) \frac{t^{n}}{n !} & \\
= & \exp \left(x w t+y(w t)^{2}\right){ }_{H} J_{0}\left(4 y \sqrt{z} t\left(\frac{x}{2 y}+w t\right), 4 z y t^{2}\right)
\end{aligned}
$$


in the 1.h.s. gives

$$
\begin{aligned}
\exp \left(u y t+v(y t)^{2}\right){ }_{H} & J_{0}\left(4 v \sqrt{x} t\left(\frac{u}{2 v}+y t\right), 4 x v^{2}\right) \\
& =\sum_{q, n=0}^{\infty} H_{q+2 n}(u, v) \frac{\left(x t^{2}\right)^{n}}{(n !)^{2}} \frac{(y t)^{q}}{q !} .
\end{aligned}
$$

(v) Taking $N=1$ and $\left\{A_{q, n}\right\}_{q, n=0}^{\infty}$ as in Remark 1.5 and using relation (1.34) in equation (2.9), we get

$$
\sum_{n=0}^{\infty} H_{n}(u, v) R_{n}(x, y) \frac{t^{n}}{(n !)^{2}}=\sum_{q, n=0}^{\infty} H_{q+n}(u, v) \frac{(y t)^{n}}{(n !)^{2}} \frac{(-x t)^{q}}{(q !)^{2}} .
$$

Now using the following bilateral generating function $[16$, p. 721]:

$$
\begin{aligned}
\sum_{n=0}^{\infty} H_{n}(x, y) R_{n} & (z, w) \frac{t^{n}}{(n !)^{2}} \\
& ={ }_{H} C_{0,0}\left(-x w t, y(w t)^{2} ; z x t, y(z t)^{2} \mid-2 w y z t^{2}\right) .
\end{aligned}
$$

in the l.h.s. of equation (4.30), we get

$$
{ }_{H} C_{0,0}\left(-u y t, v(y t)^{2} ; x u t, v(x t)^{2}-2 y v x t^{2}\right)=\sum_{q, n=0}^{\infty} H_{q+n}(u, v) \frac{(y t)^{n}}{(n !)^{2}} \frac{(-x t)^{q}}{(q !)^{2}}
$$

where ${ }_{H} C_{0,0}(\alpha, \beta ; \gamma, \delta \mid \varepsilon)$ denotes the $\left(0^{\text {th }}, 0^{\text {th }}\right)$ order of the two-index Tricomi-Bessel function defined by [16] (for $m=n=0$ )

$$
{ }_{H} C_{0,0}(x, y ; z, w \mid \tau) \sum_{r, s=0}^{\infty} \frac{(-1)^{r+s} H_{r, s}(x, y z, w \mid \tau)}{(r !)^{2}(s !)^{2}}
$$

Next, the following trilateral generating function is obtained as an application of the result (3.6):

(vi) Taking $N=1$ and $\left\{A_{q, n}\right\}_{q, n=0}^{\infty}$ as in Remark 1.1 and using relation (1.8) in equation (3.6), we get

$$
\begin{gathered}
\sum_{q=0}^{\infty} \sum_{n=0}^{\infty} \sum_{k=0}^{\left[\frac{q}{p}\right]}(\alpha)_{q} H_{q+n-p k} \\
(u, v) g_{n}^{(\alpha+q-p k, \beta)}(x, y) L_{k}\left(z_{1} z_{2}\right) \frac{\eta^{k}(-x)^{q-p k}(w t)^{q+n-p k}}{k !(q-p k) !} \\
=\exp \left(z_{2} \eta\right) C_{0}\left(-z_{1} \eta\right) \sum_{n=0}^{\infty}(\alpha)_{q} H(u, v) \frac{(y w t)^{n}}{n !}
\end{gathered}
$$

which on using relation (1.36) in the r.h.s. give the following generating functions:

$$
\begin{aligned}
& \sum_{q=0}^{\infty} \sum_{n=0}^{\infty} \sum_{k=0}^{\left[\frac{q}{p}\right]}(\alpha)_{q} H_{q+n-p k}(u, v) g_{n}^{(\alpha+q-p k, \beta)}(x, y) L_{k}\left(z_{1} z_{2}\right) \frac{\eta^{k}(-x)^{q-p k}(w t)^{q+n-p k}}{k !(q-p k) !} \\
& \quad=\exp \left(z_{2} \eta\right) C_{0}\left(-z_{1} \eta\right)(1-\mathrm{u} y w t)^{-\alpha}{ }_{2} F_{0}\left(\frac{\alpha}{2}, \frac{\alpha+1}{2} ;-; \frac{4 v(y w t)^{2}}{(1-u y w t)^{2}}\right) \cdot
\end{aligned}
$$


Similarly other trilateral generating functions can be obtained as applications of the result (3.6) with the help of Remarks 1.2-1.5

Finally, it is worthy to note that by using relation (1.38) the results obtained in this section give many bilateral and trilateral generating functions for the classical Hermite polynomials $H_{n}(x)$ associated with other polynomials.

\section{Concluding remarks}

The approach adopted in this paper is general and can be exploited to establish further consequences regarding other special polynomials. Here, we establish some generating functions involving the 2VLP $L_{n}(x, y)$ given in equations (1.28) and (1.29)

Now, following the same procedure leading to assertion (2.1) of Theorem 2.1, we get the following family of bilateral generating relation involving the $2 \mathrm{VLP} L_{n}(x, y)$ and the $2 \operatorname{VESP} S_{n}^{q, N}(x, y)$ :

$$
\sum_{q, n=0}^{\infty} L_{q+n}(u, v) S_{n}^{q, N}(x, y) \frac{t^{n} w^{q}}{q !}=\sum_{n, q=0}^{\infty} L_{q+N n}(u, v) A_{q+N n, n} \frac{\left(y \mathrm{t}^{N}\right)^{n}}{n !} \frac{(w+x t)^{q}}{q !} .
$$

Putting $w=-x t$ and $w=0$ in equation (5.1) respectively, we get

$$
\sum_{q, n=0}^{\infty} L_{q+n}(u, v) S_{n}^{q, N}(x, y) \frac{(-x)^{q} t^{n+q}}{q !}=\sum_{n=0}^{\infty} L_{N n}(u, v) A_{N n, n} \frac{\left(y t^{N}\right)^{n}}{n !}
$$

and

$$
\sum_{n=0}^{\infty} L_{n}(u, v) S_{n}^{0, N}(x, y) t^{n}=\sum_{q, n=0}^{\infty} L_{q+N n}(u, v) A_{q+N n, n} \frac{\left(y t^{N}\right)^{n}}{n !} \frac{(x t)^{q}}{q !},
$$

respectively.

Taking $\left\{A_{q, n}\right\}_{q, n=0}^{\infty}$ as in Remark 1.4 and using relation (1.23) in equation (5.2), we get

$$
\sum_{q, n=0}^{\infty} L_{q+n}(u, v)_{N} L_{n}(x, y) \frac{(-y)^{q} t^{n+q}}{n ! q !}=\sum_{n=0}^{\infty} L_{N n}(u, v) \frac{\left(x t^{N}\right)^{n}}{(n !)^{2}} .
$$

For $N=1$ and replacing $x$ by $-x$ in equation(5.4) and then using relation (1.27) and the identities

$$
t^{n} L_{n}(x, y)=L_{n}(x t, y t),
$$

we get the following bilinear generating function:

$$
\sum_{q, n=0}^{\infty} L_{q+n}(u, v) L_{n}(x, y) \frac{(-y)^{q} t^{n+q}}{n ! q !}={ }_{L} C_{0}(u x t, v x y)
$$

where ${ }_{L} C_{0}(x, y)$ denotes the $0^{\text {th }}$ order Laguerre-Tricomi functions defined by [12]

$$
{ }_{L} C_{0}(x, y)=\sum_{k=0}^{\infty} \frac{(-1)^{k} L_{k}(x, y)}{(k !)^{2}} .
$$


Again taking $\left\{A_{q, n}\right\}_{q, n=0}^{\infty}$ as in Remark 1.4 and using relation (1.23) in equation (5.3), we get

$$
\sum_{n=0}^{\infty} L_{n}(u, v)_{N} L_{n}(x, y) \frac{t^{n}}{n !}=\sum_{q, n=0}^{\infty} L_{q+N n}(u, v) \frac{\left(x t^{N}\right)^{n}}{(n !)^{2}} \frac{(y t)^{q}}{q !}
$$

which for $N=1$ and replacing $x$ by $-x$ and using equation (1.27) becomes

$$
\sum_{n=0}^{\infty} L_{n}(u, v) L_{n}(x, y) \frac{t^{n}}{n !}=\sum_{q, n=0}^{\infty} L_{q+n}(u, v) \frac{(-x t)^{n}}{(n !)^{2}} \frac{(y t)^{q}}{q !} .
$$

Now, using the following bilinear generating function [17]:

$$
\sum_{n=0}^{\infty} L_{n}(u, v) L_{n}(x, y) \frac{t^{n}}{n !}=\exp (v y t) C_{0,0}(x v t, y u t, x u t)
$$

in the 1.h.s. of equation (5.9), we get

$$
\exp (v y t) C_{0,0}(x v t, y u t, x u t)=\sum_{q, n=0}^{\infty} L_{q+n}(u, v) \frac{(-x t)^{n}}{(n !)^{2}} \frac{(y t)^{q}}{q !}
$$

where $C_{0,0}(x, y, z)$ denotes the $\left(0^{\text {th }}, 0^{\text {th }}\right)$ order 2-indext 3 -variable Tricomi functions defined by $[18]$

$$
C_{0,0}(x, y, z)=\sum_{k=0}^{\infty} C_{k}(x) C_{k}(y) \frac{z^{k}}{k !} .
$$

Remark 5.1 As in Section 4 many generating functions can be obtained as applications of results (5.2) and (5.3) with the help of Remarks 1.1-1.3 and 1.5

\section{Acknowledgements}

The authors would like to thank the referee for useful comments and suggestions.

\section{References}

[1] Srivastava, H. M. A contour integral involving Fox's H-function. Indian J. Math. 1972. 14: $1-6$.

[2] Srivastava, H. M. and Manocha, H. L. A Treatise on Generating Function. New York: Halsted Press. 1984.

[3] Gonzalez, B., Matera, J. and Srivastava, H.M. Some q-generating function and associated generalized hypergeometric polynomials. Math. Comput. Model 2001. 34: 133175.

[4] Al Gonah A. A. Some generating relations involving 2-variable Laguerre polynomials and extended Srivastava polynomials. Konuralp J. Math 2015. 3(2): 131-139.

[5] Özarslan, M. A. Some families of generating function for the extended Srivastava polynomials, Appl. Math. Comput. 2011. 218: 959-964.

[6] Altin, A., Erkus, E. and Özarslan, M.A. Families of linear generating function for polynomials in two variables. Integr. Transform. Spec. Funct 2006. 17: 315-320. 
[7] Özergin, E., Özarslan, M.A. and Srivastava, H.M. Some families of generating functions for a class of bivariate polynomials. Math. Comput. Model 2009. 50: 1113-1120.

[8] Dattoli, G., Ricci, P. E. and Cesarano, C. The Lagrange polynomials, the associated generalizations and the umbral calculus. Integr. Transforms and Spec. Func. 2003. 14(2): 181- 186.

[9] Dattoli, G. Generalized polynomials, operational identities and their applications. $J$. Comput. Appl. Math. 2000. 118(1): 111-123.

[10] Appell, P. and Kampé de Fériet J. Function hypergéométriques et hypersph-éeriques: Polynômes d'Hermite. Paris: Gauthier-Villars. 1926.

[11] Dattoli, G., Lorenzutta, S., Mancho, A.M. and Torre, A. Generalized polynomials and associated operational identities. J. Comput. Appl. Math. 1999. 108(2): 209-218.

[12] Dattoli, G. HermiteBessel and LaguerreBessel function: A by product of the monomiality principle. In: Advanced Special Function and Applications, Melfi, 1999, in: Proc. Melfi Sch. Adv. Top. Math. Phys. 2000. 1: 147-164.

[13] Dattoli, G., Ricci, P.E. and Cesarano, C. A note on Legendre polynomials. Inter. Nonlinear Sci. Numer. Simulation 2001. 2(4): 365-370.

[14] Rainville, E. D. Special Function New York: Macmillan. 1960 (reprinted by Chelsea Publ. Co., Bronx, New York). 1971.

[15] Dattoli, G., Torre, A. and Lorenzutta, S. Operational identities and properties of ordinary and generalized special function. Math. Anal. Appl. 1999. 236(2): 399-414.

[16] Dattoli, G. Bilateral generating function and operational methods. J. Math. Anal. Appl. 2002. 269: 716-725.

[17] Al Gonah, A. A. and Khan, S. New classes of Hermite-Tricomi functions and generating relations. Georgian Math. J. 2014. 21(1): 11-24.

[18] Dattoli, G., Ricci, P. E., Khomasuridze, I. and Migliorati, M. Generating functions involving arbitrary products. Tamsui Oxf. J. Math. Sci. 2007. 239(3): 269-276 